Kendal, E. (2020): Evaluation of some barley genotypes with genotype by yield* trait (GYT) biplot method. Agriculture and Forestry, 66 (2): 137-150.

\title{
Enver KENDAL $L^{1}$
}

DOI: 10.17707/AgricultForest.66.2.13

\section{EVALUATION OF SOME BARLEY GENOTYPES WITH GEOTYPE BY YIELD* TRAIT (GYT) BIPLOT METHOD}

\begin{abstract}
SUMMARY
Determination of the most appropriate genotypes based on the multiple trait index is a new method in plant breeding programs. Unpredictable climatic conditions are altering the selection of genotypes based on multiple environmental conditions and multiple traits. In barley breeding programs, some traits (quality, earliness, lodging, etc.) can serve many of our primary breeding purposes other than grain yield. For this reason, the genotype by yield*trait (GYT) biplot approach was used to definite the best barley candidate among 12 barley genotypes based on multi (three) location and multi (nine)traits. In this study, the strengths and weaknesses of each genotype were determined by combining yield and other target traits with GYT biplot method. The general adaptability of each genotype in terms of all features showed differences with concerning for the average of years. On the other hand adaptability of genotypes differed significantly in terms of GYT biplot and GT biplot methods. In the GT biplot method, both the properties and the genotypes showed a wide distribution, whereas in the GYT biplot method yield-feature combinations showed a narrower variation and the most stable genotypes were identified more clearly. Besides, it was concluded that GT biplot method GT bipot method is not very ideal for determining the best genotypes, whereas GYT biplot showed that G4 genotype, was the best; G3, G7, and G5 (Alt1kat) variety were ideal genotypes for combined traits. GYT biplot has shown that superior, ideal and stable genotypes can be detected visually by combining all traits in breeding programs.
\end{abstract}

Keywords: Barley, genotypes, multi-location, trait, GYT.

\section{INTRODUCTION}

Barley (Hordeum vulgare L.) is a very considerable crop for different industries (Animal feed, malt industries, human food, and biodiesel) and has been produced nearly 135-145 million metric tons per year after corn, wheat, and rice in the world. The production of barley, ranged between 5.5-7.5 million tons depending on the year and it is the most produced after wheat in Turkey. Today, the barley cultivated in the world, approximately $65-70 \%$ is used as animal feed, $33-35 \%$ as malt in beer, whiskey with biodiesel production and $2-3 \%$ as human

\footnotetext{
${ }^{1}$ Enver Kendal (corresponding author: enver21_1@hotmail.com), Mardin Artuklu University, Kiziltepe Vocational Training High School, Department of Crops and Animal Production, Mardin, TURKEY.

Notes: The authors declare that they have no conflicts of interest. Authorship Form signed online. 
food in food production. While in Turkey, 90-92\% of barley consumption is used as animal feed and the rest of it as malting in the brewing and food industry (Anonymous 1).

Plant breeders have been working in all fields for many years in order feed the developing world population and in recent years, they are focused on developing high-quality varieties for a healthier diet. Since there is an inverse relationship between grain yield and quality, it is very difficult to develop varieties that are both high-yielding and high-quality. In addition, many ecological and agronomic problems are encountered during breeding activities, limiting the success of plant breeders and to develop different models to overcome these problems. Evaluation of genotypes is confronted with two major problems. The first is the negative interaction between the genotype and the environmental interaction (GE) and the second, the basic traits (Kendal, 2019; Yan and Frégeau-Reid, 2018).

The GT biplot technique has been used successfully by many researchers for a long time to see the relationship between genotype by trait in different plants, and effective selections were made in breeding programs according to the interaction between genotype by trait. Despite the benefits of identifying the relationships between the traits of genotypes and trait profiles, GT biplot, cannot give enough results to the breeders about which genotype to be selected or recommended and which genotype could not be selected or eliminated. Therefore, GYT biplot technique was designed to complete the deficiencies encountered in the GT biplot technique and to enable a more efficient selection of plant breeders. GYT biplot is used to sort genotypes according to their general advantages over yield by trait combinations and to show profiles of traits (Mohammadi, 2019).

The first subjects for breeders; genotype $\mathrm{x}$ environment interactions (GEI) have been studied for many years. Many different methods (GE, GEI, AMMI, GET) have been developed to characterize the behavior of varieties under different environmental conditions. In this regard, many researchers who work with cereals in different years and environments (Kilic, 2014; Mohammadi et al., 2014; Sayar and Han, 2015), reported that the interaction of genotype $\mathrm{x}$ year $\mathrm{x}$ location (GYL) is very important, while Yan and Tinker (2006) suggested that the number of locations should be increased because the GEI is smaller than the other variance components and the genotype $\mathrm{x}$ location (GL) variance component is also large.

The second subject is to develop varieties that can give good results (high efficiency and quality, resistant to diseases and drought and temperature stress and frost) in different environmental conditions. It is very difficult to improve the best varieties in terms of all traits studied in different environments (Sayar, 2017; Kendal, 2019). The reason is that the target traits are often negatively correlated in such a way that the development in one trait usually leads to decreased levels in one or more other traits. Therefore, the barley breeders understand the nature of the correction of yield with related attributes. Some features (heading time and 
canopy temperature) are very important to know if any genotype is resistant to drought, heat stress and cold damage, plant height, lodging, i.e., and protein content, thousand-grain yield and hectoliter weight are important to improve quality of barley in Southeastern Anatolia Region of Turkey. Therefore, this study is aimed to use GYT biplot and to identify the traits associated with grain yield in barley to develop new cultivars in terms of high yield, quality, and better agronomic and physiological traits in different environmental conditions.

\section{MATERIAL AND METHODS}

Twelve spring barley genotypes including two checks (Alt1kat and Şahin 91) were evaluated in three locations during the 2011-2012 and 2012-2013 growing seasons. The information on genotypes is presented in Table 1 and about locations in Table 2.

Table 1. The code, name/pedigree, origin, and spike type of barley genotypes

\begin{tabular}{|l|l|l|c|}
\hline Code & Name of cultivar and pedigree of lines & Origin & Spike type \\
\hline G1 & $\begin{array}{l}\text { NK1272/Moroc 9-75/6/.. } \\
\text { SEA01 04-OS.0S-0SD-0SD-0SD-0SD-0SD-0SD-0SD }\end{array}$ & AARI & 2 rows \\
\hline G2 & $\begin{array}{l}\text { ROBUST//GLORIA-.. } \\
\text { CBSS00M00027S.0S-0SD-0SD-1SD-0SD--0SD-0SD-0SD }\end{array}$ & ICARDA & 6 rows \\
\hline G3 & $\begin{array}{l}\text { CABUYA/JUGL } \\
\text { CBSS00M00060S.0S-0SD-0SD-01SD-0SD-0SD-0SD-0SD }\end{array}$ & ICARDA & 6 rows \\
\hline G4 & $\begin{array}{l}\text { ARUPO/K8755//MORA/3.. } \\
\text { CBSS00M00098S.0S-0SD-0SD-1SD-0SD-0SD-0SD-0SD }\end{array}$ & ICARDA & 2 rows \\
\hline G5 & ALTIKAT(cheeck) & GAPIARTC & 6 rows \\
\hline G6 & $\begin{array}{l}\text { ARUPO/K8755//MORA/3/CERISE/SHYRI//ALELI/4/ } \\
\text { CBSS00M00098S.0S-0SD-0SD-2SD-0SD-0SD-0SD-0SD }\end{array}$ & ICARDA & 2 rows \\
\hline G7 & $\begin{array}{l}\text { ARUPO/K8755//MORA/3/CERISE/SHYRI//ALELI/4/ } \\
\text { CBSS00M00098S.0S-0SD-0SD-4SD-0SD-0SD-0SD-0SD }\end{array}$ & ICARDA & 2 rows \\
\hline G8 & $\begin{array}{l}\text { RECLA 78/SHYRI 2000 } \\
\text { CBSS00M00122S.0S-0SD-0SD-4SD-0SD-0SD-0SD-0SD }\end{array}$ & ICARDA & 2 rows \\
\hline G9 & $\begin{array}{l}\text { CUCAPAH/PUEBLA/7/ROBUST//GLORIA-BAR/COPAL } \\
\text { CBSS00M00206S.0S--0SD-0SD-5SD-0SD-0SD-0SD-0SD }\end{array}$ & ICARDA & 6 rows \\
\hline G10 & SHHIN 91(cheeck) & GAPIARTC & 2 rows \\
\hline G11 & $\begin{array}{l}\text { TAPIR-BAR/PETUNIA 1 } \\
\text { CBWS00WM00056S.0S-0SD-0SD-1SD-0SD-0SD-0SD-0SD }\end{array}$ & ICARDA & 6 rows \\
\hline G12 & UNKONOWN & AARI & 6 rows \\
\hline
\end{tabular}

G: Cultivar, ICARDA: International Center for Agricultural Research in the Dry Areas_GAPIARTC:

GAP International Agricultural Research and Training Center: AARI: Aegean Agricultural Research Institute

Table 2. Years, sites, codes and coordinate status of environment.

\begin{tabular}{|l|l|c|c|c|c|}
\hline Years & Sites & Altitude $(\mathrm{m})$ & Latitude & Longitude & $\begin{array}{c}\text { Averag. of } \\
\text { pers.(mm) }\end{array}$ \\
\hline \multirow{2}{*}{$\begin{array}{l}2012-2013 \\
2013-2014\end{array}$} & Diyarbakır & 612 & $37^{\circ} 55^{\prime} \mathrm{N}$ & $40^{\circ} 14^{\prime} \mathrm{E}$ & 483.5 \\
\cline { 2 - 6 } & Adiyaman & 685 & $37^{\circ} 46^{\prime} \mathrm{N}$ & $38^{0} 17^{\prime} \mathrm{E}$ & 704.3 \\
\cline { 2 - 6 } & Hazro & 995 & $38^{\circ} 24^{\prime} \mathrm{N}$ & $40^{\circ} 24^{\prime} \mathrm{E}$ & 891.9 \\
\hline
\end{tabular}

The trials were carried out in a randomized block design with four replications. Sowing density was used as 450 seeds per $\mathrm{m}^{-2}$. Plot size was $7.2 \mathrm{~m}^{-2}$ $(1.2 \times 6 \mathrm{~m})$ consisting of 6 rows spaced $20 \mathrm{~cm}$ apart. Sowing of trials was done in 
November in three locations and bot of year. The fertilizing percentages were used as $60 \mathrm{~kg} \mathrm{~N}$ and $\mathrm{P} \mathrm{ha}^{-1}$ with planting and $60 \mathrm{~kg} \mathrm{~N} \mathrm{ha}^{-1}$ applied to each plot at tillering. Harvesting was done using a Hege 140 harvester in an area of $6 \mathrm{~m}^{2}$ in each plot. Moreover, data on grain yield, agronomic traits (plant height, heading date), physiological traits (canopy temperatures, SPAD chlorophyll (Minolta Co. Ltd., Tokyo, Japan)) grain quality traits (protein content, seed humidity, thousand-grain weight, and hectoliter weight) were recorded for each genotype in each plot, while canopy temperature and SPAD reading only in two locations across two years.

\section{Statistical analysis (GYT and GT)}

The data of twelve barley genotypes in multi-location and multi-year trials analyzed by GT biplot method, as recommended by Yan and Thinker (2005) and, GYT biplot method, as recommended by Yan and Frégeau-Reid (2018). A superiority index (SI) combining all yield-trait integrations were calculated based on the standardized GYT (Yan and Frégeau-Reid 2018). Biplot method was built for all scored traits of genotypes using Genstat 14 release software program. The data were graphically analyzed for the interpretation of GT and GYT using the GGE biplot software. The Fig. 1(1A-1E) was produced based on the performance of each genotype for each trait (GT), the Fig. 2 (2A-2E) was generated based on the performance of genotypes by yield*traits (GYT).

\section{RESULTS AND DISCUSSION}

The Biplot of genotype by trait $(G T)$ :

The mean data of tarits across two years in three locations of 12 barley genotypes are shown in Table 3 .

Table 3. The mean data of tarits across two years in three location of 12 barley genotypes

\begin{tabular}{|c|c|c|c|c|c|c|c|c|c|}
\hline Genotype & $\begin{array}{c}\text { YLD } \\
\left(\mathrm{kg}^{-1} \mathrm{~h}^{-1}\right)\end{array}$ & $\begin{array}{c}\text { HD } \\
(\mathrm{date})\end{array}$ & $\begin{array}{c}\text { PH } \\
(\mathrm{cm})\end{array}$ & $\begin{array}{c}\text { TGW } \\
(\mathrm{g})\end{array}$ & $\begin{array}{c}\text { HW } \\
(\mathrm{kg} / \mathrm{hl})\end{array}$ & $\begin{array}{c}\text { PC } \\
(\%)\end{array}$ & $\begin{array}{c}\text { SH } \\
(\%)\end{array}$ & CT & SPAD \\
\hline 1 & 4271 & 98.1 & 84.1 & 42.0 & 73.2 & 14.4 & 7.6 & 28.7 & 45.3 \\
\hline 2 & 4419 & 96.2 & 91.9 & 38.1 & 70.3 & 12.6 & 7.7 & 28.1 & 42.6 \\
\hline 3 & 4485 & 98.2 & 85.0 & 42.8 & 70.5 & 13.3 & 7.7 & 29.0 & 43.5 \\
\hline 4 & 4910 & 96.4 & 87.2 & 43.7 & 73.0 & 12.8 & 7.7 & 27.9 & 45.0 \\
\hline Alt1kat & 4776 & 98.7 & 82.5 & 38.6 & 67.9 & 12.5 & 7.6 & 29.3 & 49.4 \\
\hline 6 & 4429 & 97.3 & 80.0 & 47.3 & 74.2 & 13.6 & 7.6 & 28.4 & 44.8 \\
\hline 7 & 4495 & 95.1 & 86.3 & 43.6 & 71.7 & 12.9 & 7.7 & 28.6 & 43.6 \\
\hline 8 & 4545 & 95.0 & 80.0 & 44.3 & 72.6 & 13.6 & 7.6 & 28.7 & 43.8 \\
\hline 9 & 3971 & 99.6 & 82.5 & 40.3 & 64.3 & 13.7 & 7.4 & 28.6 & 47.2 \\
\hline Şahin 91 & 4120 & 105.8 & 76.0 & 45.3 & 69.8 & 14.2 & 7.5 & 28.3 & 42.8 \\
\hline 11 & 4061 & 98.1 & 89.6 & 40.4 & 71.2 & 13.3 & 7.7 & 27.8 & 45.3 \\
\hline 12 & 4216 & 97.0 & 88.4 & 41.9 & 69.8 & 13.5 & 7.5 & 28.6 & 44.9 \\
\hline Mean & 4392 & 98.0 & 84.0 & 42.0 & 71.0 & 13.0 & 8.0 & 28.0 & 45.0 \\
\hline SD & 280.7 & 19.2 & 9.6 & 56.0 & 17.0 & 10.2 & 4.0 & 4.6 & 8.1 \\
\hline
\end{tabular}

YLD: yield, HD: heading date, PH: plant height, TGW: thousand grain weight, HW: hectoliter weight, PC: protein content, HS: humidity of seed, CT: canopy temperatures, SPAD: soil-plant analysis development. 
The pair-waise correlation among traits of 12 spring barley genotypes are shown in Table 4. These data were used to generated a GT biplot Fig.1, although the genotype is compatible with biplot, it represents only $62.49 \%$ of the variation.

Table 4. Pairwaise corelations among traits of 12 spring barley genotypes.

\begin{tabular}{|c|c|c|c|c|c|c|c|c|}
\hline & YLD & HD & $\mathrm{PH}$ & TGW & HW & PC & $\mathrm{SH}$ & CT \\
\hline $\mathrm{HD}$ & $-0.462 \mathrm{~ns}$ & & & & & & & \\
\hline $\mathrm{PH}$ & $0.072 \mathrm{~ns}$ & $-0.565 \mathrm{~ns}$ & & & & & & \\
\hline TGW & $0.073 \mathrm{~ns}$ & $0.117 \mathrm{~ns}$ & $-0.5983^{*}$ & & & & & \\
\hline HW & $0.387 \mathrm{~ns}$ & $-0.382 \mathrm{~ns}$ & $0.038 \mathrm{~ns}$ & $0.5818^{*}$ & & & & \\
\hline $\mathrm{PC}$ & $-0.6262 *$ & $0.475 \mathrm{~ns}$ & $-0.541 \mathrm{~ns}$ & $0.476 \mathrm{~ns}$ & $0.114 n s$ & & & \\
\hline $\mathrm{SH}$ & $0.558 \mathrm{~ns}$ & $-0.460 \mathrm{~ns}$ & $0.364 \mathrm{~ns}$ & $0.082 \mathrm{~ns}$ & $0.6643 *$ & $-0.506 n s$ & & \\
\hline CT & $0.183 \mathrm{~ns}$ & $-0.004 n s$ & $-0.359 \mathrm{~ns}$ & $-0.113 n s$ & $-0.315 \mathrm{~ns}$ & $-0.021 \mathrm{~ns}$ & $-0.308 \mathrm{~ns}$ & \\
\hline SPAD & $0.122 \mathrm{~ns}$ & $0.021 \mathrm{~ns}$ & $-0.084 n s$ & $-0.413 n s$ & $-0.470 \mathrm{~ns}$ & $-0.196 n s$ & $-0.282 \mathrm{~ns}$ & $0.433 \mathrm{~ns}$ \\
\hline
\end{tabular}

$*$ Value significant for 0.05 probability level. ns: not significant

The Fig. 1(A) visualize the relationships between properties and trait by genotypes profiles. A biplot such a graph to be interpreted bi-directionally has the following comments (Yan et al., 2000; Yan and Tinker, 2006). The cosine of the angle between the vectors of the two properties approaches the Pearson correlation between them. Therefore, an angle of less than $90^{\circ}$ shows a positive correlation, an angle greater than $90^{\circ}$ shows a negative correlation and an angle of $90^{\circ}$ shows zero correlation. If the vector of a trait is longer than other vectors, the variation of this trait on genotypes is higher than the other traits, if the vector length of any trait is very short than other traits vector then the variation of this trait is very low. The angle between the vector of any genotype and any trait gives information about the state of the genotypes. If the angle is quite sharp and narrow, it indicates that the genotype is below average for that trait if the angle is too large then the genotype is under of mean data of traits. The length of the vector of a genotype indicates the strength or weakness of the genotype for all trait profiles. Depending upon these principles described in the GT biplot technique, the following observations were made about Fig. 1(A). Considering the observations on this figure indicated that grain yield was positively correlated with $(\mathrm{PH}, \mathrm{SH}, \mathrm{HW})$, while negatively correlated with quality traits (HD, PC, TGW) and it was not associated with physiological traits (CT and SPAD). On the other hand, the explanations are confirmed by the correlation values in (Table 2).

The Fig.1(B) visualized the stability of genotypes based on traits, A vertical mean axis, and a horizontal stability axis are created over the average values and the genotypes are evaluated according to these axes'. If the genotypes are located below the verticle axis, they are unpreferable if they are located above the verticle axis, they are preferable genotypes. On the other hand; if the genotypes are located near or center of the horizontal line, they are stable, and if they are located away from the horizontal line, they are unstable (Kendal and Sayar, 2016; Yan and Rajcan, 2002). Considering the Fig.1(B) with this prediction; the G3 is quite stable because this genotype is located at the center of the horizontal axis, and G8 is stable because this genotype is located near center of horizontal axis; G6 and G9 are unstable, because they are located far from the 
center of the horizontal axis. While, G12, G9, and G5(control) are unpredictable genotypes because they were located under the vertical axis line, other genotypes (G4, G6, G7 and G8), in which located above on-axis vertical line, are preferable genotypes based on trait profiles.

The Fig.1(C) visualized the discriminating and representativeness of genotypes based on traits, and provided a representative "ideal center" over the mean values of the properties and offers the opportunity to evaluate genotypes according to their proximity or distance from this center(Yan and Tinker, 2005; Oral, 2018. If the genotypes are located in the center, they are the most ideal, if they are located upon the average perpendicular axis, but far from the center, it means that they are ideal, if they are located below perpendicular axis (red tik line), it means that they are undesirable.
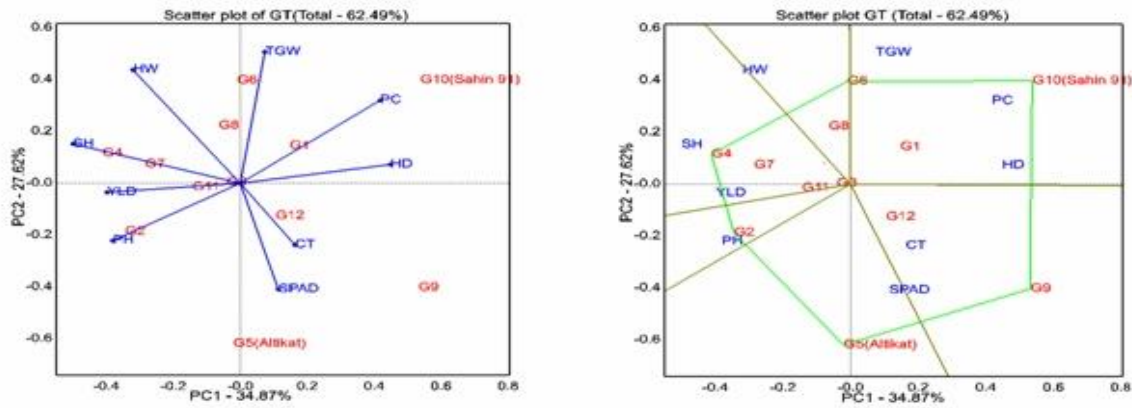

(1A)

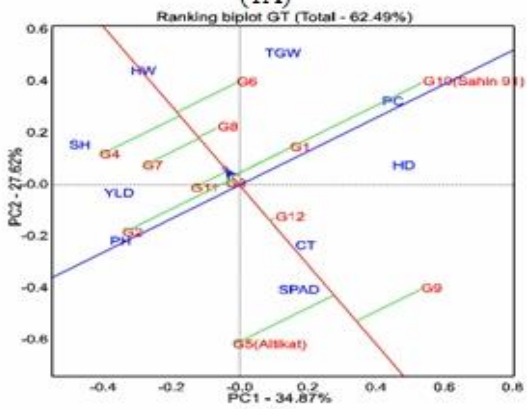

(1D)

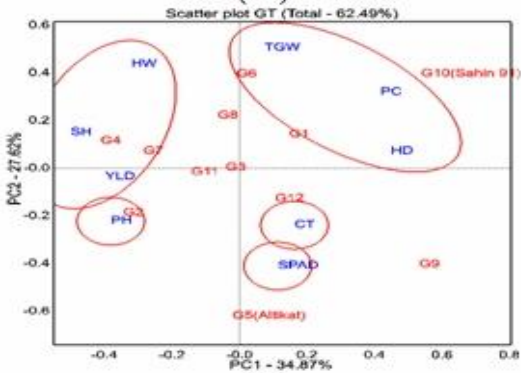

(1E)

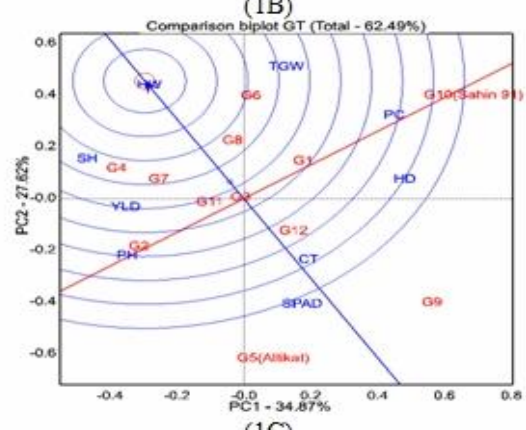

(1C)

Figure 1. Genotype by trait values across two years (Table 3 and Table 4).

(1A) the relation of GT based two seasons data, (1B) the stability of GT based two seasons data, (1C) the comparison of GT based on two years data, (1D) which-won-where/what of GT biplot based on across season data. (1E) the group of GT based on two years data. 
Considering the Fig.1(C) with this prediction; G6 is more ideal than G4, G7 and G8, because it is nearest to the "ideal center", while G5(control) and G9 located under perpendicular axis, and also far from "ideal center, so this two genotype are undesirable.

The Fig.1(D) visualized the polygon of which-won-where/what of GT biplot based on across season data. The figure divided by thick axis from center, and each zone separated by two thick lines is referred to as the "sector" and is indicated by numbers $1,2,3$, etc., starting from the lower right part of the graph, and if the genotypes and traits are located in the same sector, they are very close to each other (Yan and Tinker, 2006; Kendal and Sayar, 2016). Considering Fig.1(D) with this prediction; the figure is divided into 6 sectors (seperated each other by a tik line in the figure) and different traits are associated with different genotypes in each sector. The genotype G9 is a winner of the sector 1 located in the same sector with G12 and correlated to CT trait, G10 Şahin(control) is a winner of sector 2 located in the same sector with G1 with HD, PC, TGW. The genotype G6 is winning of sector 3 located in the same sector with G8 and did not correlate to any trait. The genotype G4 is winning of sector 4 located in same sector with G3, G7, and G11, YLD, SH, and HW. The genotype 2 is a winner of the sector 5 and correlated with $\mathrm{PH}$, while G5 Alt1kat(control) variety is a winner of the sector 6 with SPAD only.

The Fig.1(E) visualized the group of GT based on across season data and in the figure, the traits and genotypes have relationship, If they are located in the center a circle, it means that there is positive correlation among them (Kendal et al., 2016; Kizilgeci et al., 2019). Considering Fig.1(E) In the light of these explanations; traits were separated into 5 different groups (each one group identified by a circle). The first group was included HD, PC, TGW, the second group included GY, SH, HW, while $\mathrm{PH}, \mathrm{BT}$, and SPAD were included independent groups (3, 4 and 5). The G6 is located in group 1(HD, PC, TGW), G4 located in center group 2 (GY, SH, HW) and G2 located in the group of PH. The results showed that the G6 is a winner for HD, PC, TGW, G4 for GY, SH, $\mathrm{HW}$, and $\mathrm{G} 2$ for $\mathrm{PH}$.

The Biplot of genotype by yield trait combination (GYT):

The genotype by yield*trait (GYT) data for 12 spring barley genotypes across two years in three locations shown in (Table 5). The data in the GYT table (Table 5) was generated from the GT table (Table 3) and in GYT table, the data in each column consists of a combination of yield-trait. The standardized genotype by yield*trait (GYT) data and superiority index for 12 spring barley genotypes across two years in three locations shown in Table 6 . The genotypes were quite compatible with biplot, they represent $88.94 \%$ of the total variation (PC1 \%76.40, PC2 \%12.54). GYT biplot, in the combination with the yield and any trait, is used to measure how the grain yield is combined with that trait in genotypes. When both the grain yield and the values of any trait are low or high, the values will be either low or high and the genotypes will be evaluated accordingly. On the other hand, the GYT biplot technique was developed to determine where the value of a trait of any genotype is low, grain yield is high or 
vice versa, whether the results are affected by the combination or is there any change in the ranking of genotypes. As a result, when the values of the traits and the yield values enter the combination, the data changes and the ranking of the genotype changes. Therefore, in the GYT table, a greater value is always desirable. As mentioned above, before the interpretation of the GT biplot shapes, each figure is described in detail. These explanations cover the forms that form with GYT biplot. For this reason, GYT biplot will not be described again, but only the results obtained from only GYT biplot shapes are given below.

Table 5 . Genotype by yield*trait data for 12 barley genotypes across two years in three locations.

\begin{tabular}{|c|c|c|c|c|c|c|c|c|}
\hline Genotype & YLD*HD & YLD*PH & YLD*TGW & YLD*HW & YLD*PC & YLD*SH & YLD*CT & YLD*SPAD \\
\hline 1 & 418825 & 359031 & 179517 & 312507 & 61513 & 32378 & 122444 & 193455 \\
\hline 2 & 425053 & 405996 & 168163 & 310805 & 55764 & 33886 & 124202 & 188360 \\
\hline 3 & 440371 & 381225 & 191846 & 316232 & 59503 & 34314 & 130093 & 195008 \\
\hline 4 & 473201 & 428091 & 214516 & 358362 & 63075 & 37832 & 136928 & 220864 \\
\hline Alt1kat & 471332 & 394020 & 184258 & 324205 & 59509 & 36377 & 139698 & 235767 \\
\hline 6 & 430997 & 354320 & 209335 & 328600 & 60360 & 33849 & 125922 & 198463 \\
\hline 7 & 427306 & 387694 & 195759 & 322350 & 58030 & 34694 & 128417 & 195881 \\
\hline 8 & 431775 & 363600 & 201279 & 329840 & 61810 & 34663 & 130214 & 198980 \\
\hline 9 & 395363 & 327608 & 159930 & 255464 & 54512 & 29362 & 113620 & 187292 \\
\hline Şahin 91 & 435690 & 313120 & 186487 & 287652 & 58368 & 31024 & 116467 & 176223 \\
\hline 11 & 398486 & 363967 & 164017 & 289243 & 54059 & 31368 & 113073 & 183831 \\
\hline 12 & 408952 & 372853 & 176842 & 294449 & 56795 & 31515 & 120446 & 189098 \\
\hline Mean & 429779 & 370960 & 185996 & 310809 & 58608 & 33438 & 125127 & 196935 \\
\hline
\end{tabular}

Table 6. Standardized genotype by yield*trait data and superiority index for 12 barley genotypes across two years in three locations.

\begin{tabular}{|c|c|c|c|c|c|c|c|c|c|}
\hline Genotype & YLD*HD & YLD*PH & YLD*TGW & YLD*HW & YLD*PC & YLD*SH & YLD*CT & YLD*SPAD & $\begin{array}{c}\text { Mean } \\
\text { (SI) }\end{array}$ \\
\hline 1 & 0.97 & 0.97 & 0.97 & 1.01 & 1.05 & 0.97 & 0.98 & 0.98 & 0.99 \\
\hline 2 & 0.99 & 1.09 & 0.90 & 1.00 & 0.95 & 1.01 & 0.99 & 0.96 & 0.99 \\
\hline 3 & 1.02 & 1.03 & 1.03 & 1.02 & 1.02 & 1.03 & 1.04 & 0.99 & 1.02 \\
\hline 4 & 1.10 & 1.15 & 1.15 & 1.15 & 1.08 & 1.13 & 1.09 & 1.12 & $\mathbf{1 . 1 2}$ \\
\hline Alt1kat & 1.10 & 1.06 & 0.99 & 1.04 & 1.02 & 1.09 & 1.12 & 1.20 & 1.08 \\
\hline 6 & 1.00 & 0.96 & 1.13 & 1.06 & 1.03 & 1.01 & 1.01 & 1.01 & 1.02 \\
\hline 7 & 0.99 & 1.05 & 1.05 & 1.04 & 0.99 & 1.04 & 1.03 & 0.99 & 1.02 \\
\hline 8 & 1.00 & 0.98 & 1.08 & 1.06 & 1.05 & 1.04 & 1.04 & 1.01 & 1.03 \\
\hline 9 & 0.92 & 0.88 & 0.86 & 0.82 & 0.93 & 0.88 & 0.91 & 0.95 & $\mathbf{0 . 8 9}$ \\
\hline Şahin 91 & 1.01 & 0.84 & 1.00 & 0.93 & 1.00 & 0.93 & 0.93 & 0.89 & 0.94 \\
\hline 11 & 0.93 & 0.98 & 0.88 & 0.93 & 0.92 & 0.94 & 0.90 & 0.93 & 0.93 \\
\hline 12 & 0.95 & 1.01 & 0.95 & 0.95 & 0.97 & 0.94 & 0.96 & 0.96 & 0.96 \\
\hline SD & 1.00 & 1.00 & 1.00 & 1.00 & 1.00 & 1.00 & 1.00 & 1.00 & 1.00 \\
\hline
\end{tabular}


Based on these principles described in the GYT biplot technique, the following observations were made about relationships between yield trait combinations. Considering the above-mentioned observations was indicated that all yield-trait combinations tend to correlate positively with each other because they have yielded as a component, shown by the triangular angles between the vectors Fig.2 (A). This is an important feature of the GYT biplot (Fig.2) technique, in contrast to the GT biplot (Fig. 1); in this way, the graphical representation provides the opportunity for genotypes to be ranking in a more meaningful way. Although there is high correlation between traits in the GT, there is poor correlation between them in the GYT. For an exam, there is a positive correlation between YLD and $\mathrm{PH}$ and the negative correlation between YLD and PC and HW (Fig. 1A and Table 3). In GYT biplot technique, the same correlation can still be seen, as indicated with lower correlation values and a narrow angles between YLD * PH, YLD * PC and YLD * HW.

The effect on GYT to stability and superiority of genotypes is presented in Fig 2 (B). The horizontal line with one arrow indicates the stability line of combination and evaluate the genotypes based on this line. On the other hand, the superiority of genotypes is determined by the vertical line without an arrow. Because of these explanations, the stability and superiority analysis indicated that G4 is the most stable and superior, G3 is stable and superior, G5, G6, G7, and G8 are only superior genotypes. Moreover, the G1, G2, G9, G10, G11 and G12 are both unstable and unfavorable genotypes because they took place under the mean line of multiply traits. The superiority index (SI) ranked genotypes by mean of all traits. High values of SI (1.12) indicated the best genotypes (G4), low values of SI (0.89) indicated the poor genotypes (Fig 2B-Table 6).

Discriminating and representativeness of genotypes based on GYT combination are presented in Fig.2 (C) and provides a representative "ideal center" over the mean values of GYT. Considering the Fig.2(C) with this prediction; G4 is the ideal genotype, because it was located nearest to the "ideal center" and G3, G5, G6, G7, and G8 are desirable for GYT combination because they were located upon mean of data combination (shown as perpendicular red line). While the G1, G2, G9, G10, G11, and G12 are undesirable genotypes because these genotypes are located under mean values of vertical line.

Demonstration of trait profiles of genotypes by sector analysis "whichwon-where" in the GYT biplot can be seen in Fig.2D. The most effective genotype associated with trait profiles in each sector is indicated by a polygon peak. In the sector analysis, the figure was divided into 7 sectors. Each one sector separated eachother by two tik line and started to number from $\mathrm{x}$ coordinate $(0.0)$ and circled from right, numbered according to y coordinate. All combinations except YLD*PH were in the same sector. While G5 (Alt1kat (control) and G7 located in the same sector with YLD*PH combining, G3 and G4 are in the sector where other combinations (YLD*PH, YLD*PC, YLD*TGW, YLD*SH, YLD* $\mathrm{HW}, \mathrm{YLD} * \mathrm{CT}, \mathrm{YLD} * \mathrm{SPAD}, \mathrm{YLD} * \mathrm{HT}$ ) are present and $\mathrm{G} 4$ is also located at the vertex of the polygon in this sector. It was found that G4 was the best in 
combining all traits with YLD except PH. Other genotypes were separated from the other five sectors where trait combinations were not included. It indicated that eight genotypes did not produce a good results of combining trait, except G3, G4, G5, and G7.
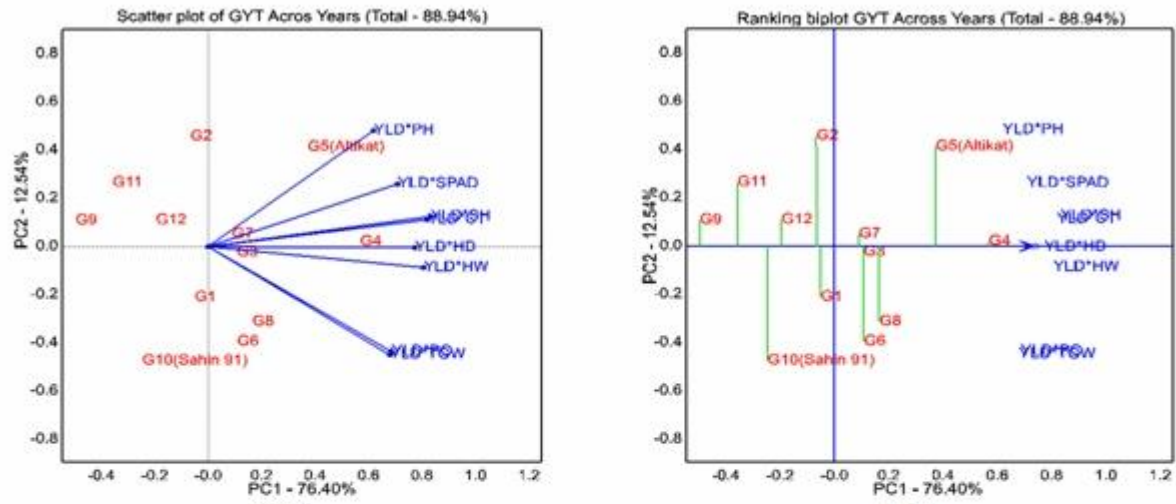

(2A)

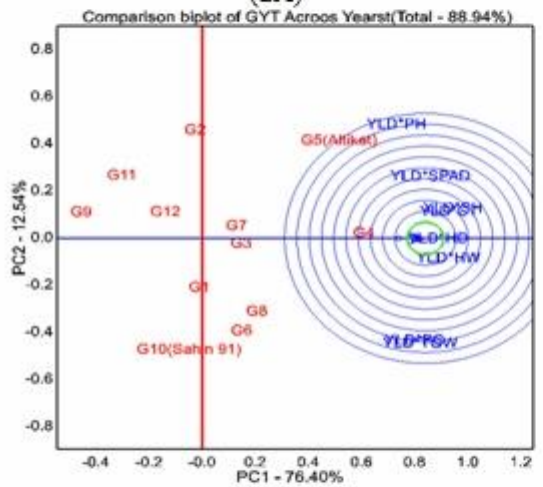

(2C)
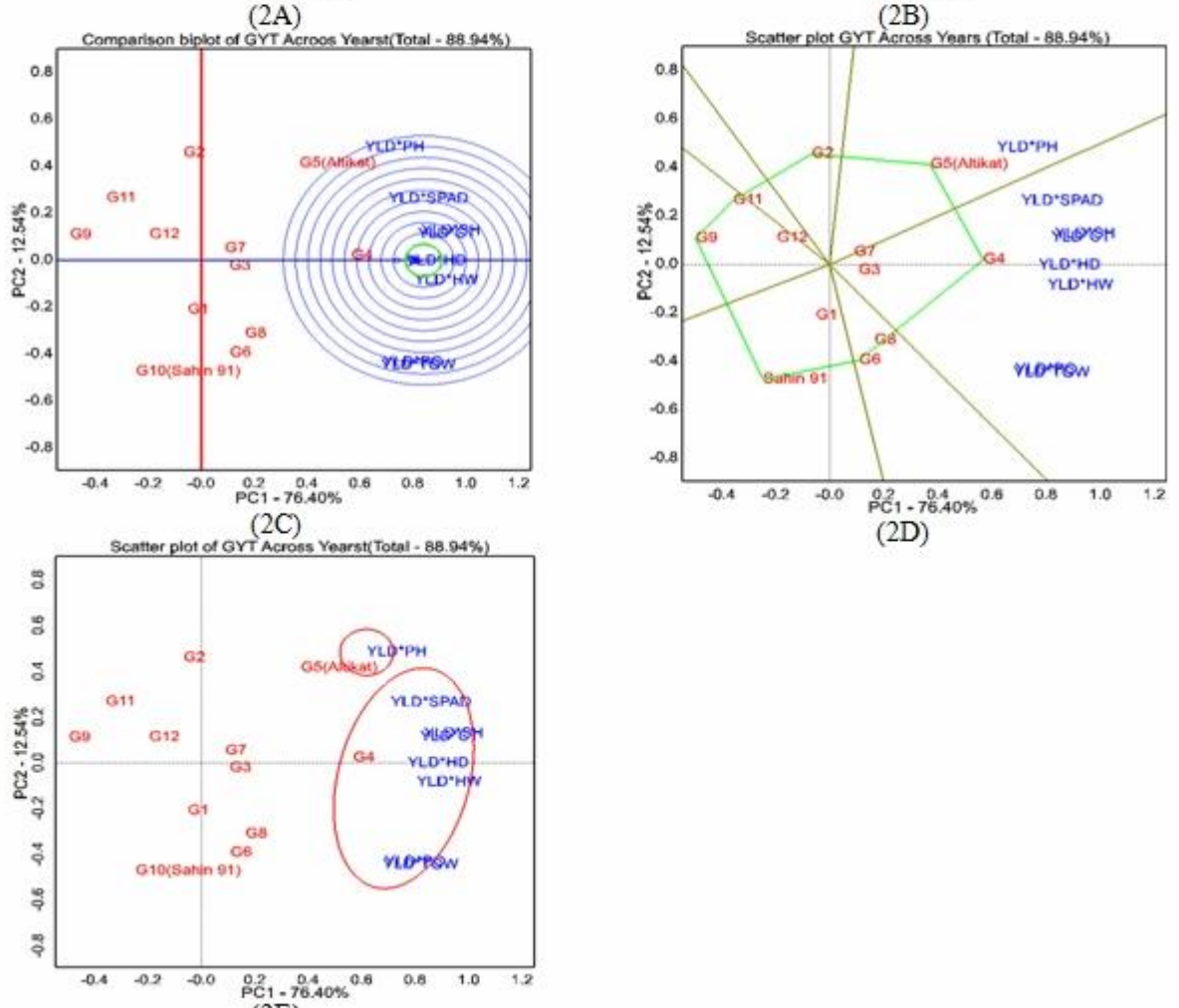

(2D)

(2E)

Figure 2. Genotype by yield*trait values across two years (Table 5 and Table 6). (2A), the relation of GYT biplot based on combination of two seasons data, (2B) the stability of GYT based on combination of two seasons data, (2C) the comparison of GYT based on combination of two seasons data, (2D) which-won-where/what of GYT based on across season data. (2E) the grup of GYT based on across locations data. 
Fig.2 (E) visualized yield-trait combinations, which are in a close relationship, located in the same circle. Considering Fig.1 (E) in the light of these explanations; yield-trait combinations were separated 2 groups. The first group were included all combinations with yield (HD, PC, TGW, GY, SH, HW PH, BT, SPAD) except YLD*PH. It indicated that there was a high correlation among all traits with yield combination except $\mathrm{PH}$. On the other hand, the figure showed that G4 was located in the center group of yield-trait combination without YLD*PH.

Since nearly 20 years, many studies have been conducted on GE, GEI and GT in different plants and the results of these studies have been published by many breeders (Dehghani et al., 2006; Yan and Tinker, 2006; Sayar, 2017; Karaman, 2019; Kizilgeci et al., 2019). However, there are almost no publications related to the evaluation of genotypes based on multiple traits (de Oliveira et al., 2019; Kendal, 2019; Yan and Frégeau-Reid, 2018). When the genotypes are evaluated for each trait separately or if the traits in each location are evaluated separately, sometime, some tricks or general effects may be missed. Therefore, breeders use different methods in breeding studies to make a calculation based on the rating system based on the effect of each trait and try to select the best genotypes. However, since the varieties registered are not registered with a selection based on the multi-feature combination of all locations, they cannot perform well due to the problem of agronomic properties, when they grow in other regions with similar conditions outside the central region. However, when the varieties are registered with a selection based on the combination of properties obtained from multiple locations with yield, then they will be quite stable in terms of all properties and yield for all similar regions. For this purpose; GYT biplot methodology has been recently developed and has been used by a few researchers for the evaluation of the data obtained from the combination of the multiple traits with yield and multiple locations in the breeding studies. GYT biplot approach has been reported to be a comprehensive and effective method since it classifies genotypes according to their levels in combination with target characteristics and graphically ranks the genotypes with their strengths and weaknesses and in different plants (Yan et al., 2019). If the selection of genotypes is based on one trait, it can be neglected in terms of other traits; therefore, it is more advantageous to use GYT biplot instead of GT biplot in breeding studies. In fact, in barley breeding studies, the yield is the only trait that can determine the effectiveness of a genotype alone; other traits (agronomic characteristics, quality characteristics or stress resistance) are valuable only for the breeders when combined with high yield levels, and these properties alone do not mean anything to growers. For example; a barley genotype is not valuable for breeders if it is high quality, resistant to temperature stress and the yield is low. However, the genotype is valuable if the genotype is both high yielding, and has good agronomic and quality characteristics as well as. Kilic et al., (2018), reported that GT biplot analysis permitted a meaningful and useful summary of GT interaction data and assisted in examining the natural relationships and variations in 
genotype performance on traits. Therefore, in selecting the best genotypes, the combined effects of yield-trait are more meaningful than the effects of individual traits. In the GT biplot technique, a great value (Table 3, Fig 1B) makes the ATC appearance insignificant in some cases (Solonechnyi et al., 2018), while in the GYT biplot technique it makes the ATC appearance a meaningful and effective tool because it ranks genotypes based on various yield-trait combinations and indicates the strengths and weaknesses of genotypes (Fig. 2(B), Table 5). The GT biplot technique was used to construct Fig. 1 (A-E) using the data in Table 3, while the GYT biplot technique was used in Fig.2 (A-E) using the data given in Table 5 and genotypes were examined with different graphs according to both techniques. While the barley producers strive to obtain maximum and highquality products from the unit area (Kendal and Dogan, 2015). Feed industrialists also strive to obtain feeds that are easy to process and demand animal breeders. All these needs can only be achieved by using GYT biplot methodology and the products which are widely used in production areas. The genotypes were examined depend on the superiority index (SI) and yield-trait combination (GYT) and the result of Fig. 2 showed that the genotypes can be evaluate than GT biplot in Fig 1.On the other hand, in GT biplot there is not clear of best genotype which is very stable for all traits, while the G4 is stable and G3and G7 for all trait in GYT biplot. Therefore, it was found in this study that GYT biplot technique is a suitable method for determining the most suitable genotype for all properties in barley breeding studies.

\section{CONCLUSIONS}

The objectives of genotypes by yield ${ }^{x}$ triats combination suggested that there are more reason to use this method in multi-location, multi-years with multi-traits studies. In GYT biplot technique, the total ratio of PC1 and PC2 in total variation is higher than GT biplot technique. In GYT biplot technique, it is seen that there is a special variation relationship between all traits and yield, while general relationship in GT biplot technique. In terms of all traits, the GYT biplot technique provides information on the general adaptability of genotypes, while the GT biplot technique provides information on specific adaptability capabilities. In terms of all traits, the stability of the genotypes and the best genotype is clearly seen in the GYT biplot technique (G4), while the GT biplot technique is more complex.

\section{REFERENCES}

Anonymus 1. http://www.fao.org/faostat/en/\#data/QC

Oliveira TRA, Amaral Gravina G, Moura Rocha M, Alcântara Neto F, Cruz DP, Oliveira GHF., Rocha RS. 2019. GYT Biplot Analysis: A New Approach for Cowpea Line Selection. Journal of Experimental Agriculture International, 41(5), 1-9.

Dehghani H, Ebadi A, Yousefi A. 2006. Biplot analysis of genotype by environment interaction for barley yield in Iran. Agronomy Journal, 98(2), 388-393. 
Karaman M. 2019. Evaluation of bread wheat genotypes in irrigated and rainfed conditions using biplot analysis. Applied Ecology and Environmental Res., 17(1), 1431-1450.

Kendal E and Sayar M.S. 2016. The stability of some spring triticale genotypes using biplot analysis, The Journal of Animal \& Plant Sciences, 26(3): 2016, Page:754765.

Kendal E. 2019. Comparing durum wheat cultivars with genotype $\times$ yield $\times$ trait $(G Y T)$ and genotype $\times$ trait (GT) by biplot method. Chilean Journal of Agricultural Research, 79(04), 512-522.

Kendal E, Sayar MS, Tekdal S, Aktas H and Karaman M. 2016. Assessment of the impact of ecological factors on yield and quality parameters in triticale using GGE biplot and AMMI analysis. Pak. J. Bot., 48(5): 1903-1913.

Kendal E, Tekdal S, \& Karaman M. 2019. Proficiency of biplot methods (AMMI and GGE) in the appraisal of triticale genotypes in multiple environments. Applied Ecology and Environmental Research, 17(3), 5995-6007.

Kendal, E., \& Dogan, Y. 2015. Stability of a candidate and cultivars (Hordeum vulgare L) by GGE biplot analysis of multi-environment yield trial in spring barley. Agriculture \& Forestry, 61(4), 307-318.

Kılıç H., Kendal E., Aktaş H. 2018 Evaluatıon of yield and some quality characters of winter barley (Hordeum vulgare L.) genotypes using biplot analysis. Agriculture \& Forestry, 64(3) 101-111.

Kilic H. 2014. Additive main effect and multiplicative interactions (AMMI) Analysis of grain yield in barley genotypes across environments, J. Agr. Sc. 20,337-344.

Kizilgeci F, Albayrak O, \& Yildirim M. 2019. Evaluation of thirteen durum wheat (Triticium durum Desf.) genotypes suitable for multiple environments using GGE biplot analysis. Fresenius Environmental Bulletin, 28(9), 6873-6882.

Kizilgeci F, Albayrak O, Yildirim M, \& Akinci C. 2019. Stability evaluation of bread wheat genotypes under varying environments by AMMI model. Fresenius Env. Bulletin, 28(9), 6865-6872.

Mohammadi R. 2019. Genotype by Yield* Trait Biplot for Genotype Evaluation and Trait Profiles in Durum Wheat. Cereal Research Communications, 47(3), 541-551.

Mohammadi R, Haghparast R, Sadeghzadeh B, Ahmadi H, Solimani K, Amri. A. 2014. Adaptation patterns and yield stability of durum wheat landraces to highland cold rainfed areas of Iran. Crop Science 54: 944-954.

Oral E. 2018. Effect of nitrogen fertilization levels on grain y1eld and yield components in triticale based on AMMI and GGE biplot analysis. Applied Ecology and Environmental Research, 16(4), 4865-4878.

Sayar M.S. 2017. Additive Main Effects and Multiplicative Interactions (AMMI) Analysis for Fresh Forage Yield in Common Vetch (Vicia sativa L.) Genotypes. Agr. \& For., 63 (1): 119-127.

Sayar MS and Han Y. 2015. Determination of seed yield and yield components of grasspea (Lathyrus sativus L.) lines and evaluations using GGE Biplot analysis method. Tarim Bilimleri Dergisi- J. Agric. Sci,, 21(1): 78-92.

Solonechnyi, P., Kozachenko, M., Vasko, N., Gudzenko, V., Ishenko, V., Kozelets, G., Usova, N., Logvinenko Y., Vinyukov, A. 2018: AMMI and GGE biplot analysis of yield performance of spring barley (Hordeum vulgare L.) varieties in multi environment trials. Agriculture and Forestry, 64 (1): 121-132. DOI:10.17707/AgricultForest.64.1.15 
Yan W, Tinker NA. 2005. An integrated biplot analysis system for displaying, interpreting, and exploring genotypex environment interaction. Crop Science, 45(3), 1004-1016.

Yan W, Tinker NA. 2006. Biplot analysis of multi-environment trial data: Principles and applications. Canadian journal of plant science, 86(3), 623-645.

Yan W, Frégeau-Reid J. 2018. Genotype by Yield* Trait (GYT) Biplot: a Novel Approach for Genotype Selection based on Multiple Traits. Sci Rep., 8:1-10.

Yan W, and Rajcan I.R. 2002. Biplot analysis of test sites and trait relations of soybean in Ontario. Canadian Journal Plant Science. 42:11-20.

Yan W, Frégeau-Reid, J, Mountain N, \& Kobler J. 2019. Genotype and management evaluation based on genotype by yield* trait (GYT) analysis. Crop Breeding, Genetics and Genomics, 1(2).

Yan W, Hunt LA, Sheng Q, Szlavnics Z. 2000. Cultivar evaluation and megaenvironment investigation based on the GGE biplot. Crop Science, 40(3), 597-605. 